\title{
Restaurations coronoradiculaires des dents dépulpées par endocouronnes
}

\section{Coronadicular restorations of teeth pulled} by endocourons

\section{MOTS-CLEFS :}

- Endocouronne, CFAO, prothèse, dent dépulpée, céramique dentaire, composite

\section{KEYWORDS:}

- Endocrown, CAD/CAM, Dental Porcelain, Dental Prosthesis Design, Root Canal Therapy, Tooth, non vital/therapy, Composite Resins

$\operatorname{AOS} n^{\circ} 283-2017$

\section{Résumé}

La restauration des molaires dépulpées constitue un défi quotidien pour le praticien. Compte tenu de la diversité des situations cliniques, des caractéristiques mécaniques de la dent dépulpée et des propositions thérapeutiques s'offrant aujourd'hui au praticien, il est difficile d'obtenir un consensus large sur les méthodes et conduites à suivre concernant la restauration de la molaire dépulpée.

Avec les progrès de l'adhésion et l'apport des matériaux esthétiques récents ainsi que l'apparition de la conception et fabrication assistée par ordinateur (CFAO), les concepts d'invasivité minimale, de gradient thérapeutique et de biomimétique tendent à être intégrés dans notre pratique quotidienne avec des restaurations prothétiques plus économes en tissus dentaires. La réalisation d'endocouronnes par CFAO directe sur molaires dépulpées répond aux exigences et progrès apportés par ces trois concepts. Les endocouronnes sont des éléments prothétiques indiqués dans la restauration des dents dépulpées. Elles consistent en une couronne monobloc avec une partie interne colonisant la chambre pulpaire, sans ancrage radiculaire.

L'objectif de ce travail est de présenter l'endocouronne au travers de quatre cas cliniques réalisées par CFAO directe utilisant 3 différents matériaux de restauration, dans le respect des principes de préservation tissulaire et de biomimétique, en tenant compte des données actuelles sur la restauration des molaires dépulpées.
Abstract

Restoration of endodontically treated molars is a daily challenge for the practitioner. Given the diversity of clinical cases, the mechanical characteristics of the non vital tooth and the therapeutic proposals the practitioner can take advantage of, it is hard to reach broad consensus on knowing which methods and behaviors to adopt towards the restoration of non vital molars. Thanks to the progress of adhesion, the contribution of recent aesthetic materials and the appearance of CAD/CAM, the concepts of minimal invasiveness, therapeutic gradient and biomimicry tend to be embedded in our daily practice through more efficient prosthetic restorations in dental tissues. Realizing endocrowns on endodontically treated molars by direct CAD/CAM meets the requirement and the progress provided by these three concepts. Endocrowns are prosthetic components used in the restoration of non vital teeth. They consist of a single-block crown with an inner portion integrated into the pulp chamber without intraradicular anchorage. This study aims to display the endocrowns carried out by direct CAD/CAM through clinical cases, using three different materials, according to the concepts of minimal invasiveness and biomimicry and to current data on the restoration of endodontically treated molars. 


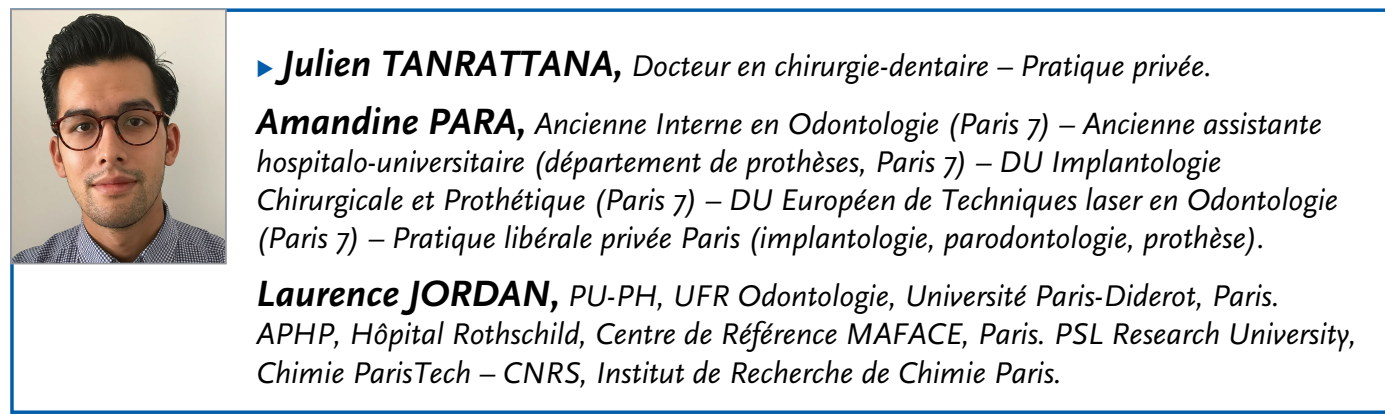

\section{INTRODUCTION}

La restauration des dents traitées endodontiquement constitue un défi quotidien pour le praticien. Compte tenu de la diversité des situations cliniques, des caractéristiques mécaniques de la dent dépulpée et des propositions thérapeutiques s'offrant aujourd'hui au praticien, il est difficile d'obtenir un consensus large sur les méthodes et conduites à suivre concernant la restauration de la dent dépulpée. Les concepts d'invasivité minimale, d'adhésion et de gradient thérapeutique sont aujourd'hui intégrés dans notre pratique quotidienne. La réalisation d'endocouronnes par Conception et Fabrication Assistée par Ordinateur (CFAO) directe répond aux exigences et progrès apportés par ces trois concepts et constitue une alternative thérapeutique de choix dans le traitement des molaires dépulpées.

\section{PLACE DE L'ENDOCOURONNE DANS UNE APPROCHE CONSERVATRICE ET BIOMIMÉTIQUE DE LA DENT DÉPULPÉE}

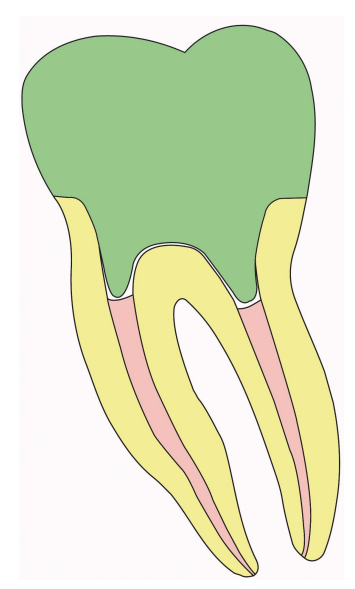

Fig. 1 :

Dessin schématique d'une molaire mandibulaire traitée endodontiquement et restaurée avec une endocouronne par CFAO.
L'endocouronne est une coiffe prothétique monobloc à ancrage caméral avec absence d'ancrage radiculaire. Son développement récent va de pair avec l'évolution des concepts d'économie tissulaire et d'invasivité minimale. Les systèmes CFAO ont également accéléré le développement des techniques adhésives, dont les endocouronnes font parti intégrantes. La préparation pour endocouronne a l'avantage d'être très conservatrice des tissus résiduels en favorisant le collage par rapport aux principes de rétention macro-mécanique (1) (2). L'extension camérale prenant appui sur la chambre pulpaire recouvre l'ensemble des faces axiales de manière plus ou moins totale (3). De cette façon, l'endocouronne associe sur une même pièce prothétique la portion de reconstruction coronaire et la portion de comblement de la chambre pulpaire. Le respect des structures biologiques se traduit par l'absence de préparation canalaire et de tenon radiculaire, évitant ainsi le risque de fragilisation de la racine pouvant entrainer fêlures et même perforations si la préparation canalaire est mal conduite. L'endocouronne permet également un renforcement des tissus sains résiduels grâce au collage et au maintien fonctionnel de la dent sur l'arcade (4). En outre, cette technique présente l'avantage d'augmenter la résistance de la pièce prothétique du fait d'une plus grande épaisseur de matériau. Le concept de l'endocouronne, conservant l'intégrité structurelle des racines, permet une possible réintervention vers les racines préservées, bien qu'elle n'en soit pas la première finalité.

La surface de collage offerte par la chambre pulpaire et la portion coronaire des canaux radiculaires est souvent égale voire supérieure à celle obtenue lors du collage d'un ancrage radiculaire de $8 \mathrm{~mm}$ de profondeur. L'application des résines adhésives et leur polymérisation est en outre plus aisé et bien mieux contrôlable dans ces conditions plutôt qu'à $8 \mathrm{~mm}$ de profondeur dans le canal radiculaire (5). L'inspection directe de la dentine est plus aisée, tout comme le contrôle de l'humidité et la présence éventuelle d'acide de mordançage résiduel. L'endocouronne permet donc une préservation des tissus résiduels de la dent dépulpée, en tirant au maximum profit de la surface de collage offerte par la dent. Les concepts d'invasivité minimale et d'adhésion sont désormais intégrés dans notre pratique quotidienne, nous donnant les moyens de préserver au maximum les tissus dentaires. Il nous est permis aujourd'hui de voir encore plus loin avec le concept de «biomimétique ». Si dans le domaine scientifique, la biomimétique implique la reproduction ou la copie d'un modèle ou d'une référence (6) (7), la biomimétique appliquée en odontologie permet de restaurer biologiquement, biomécaniquement et esthétiquement les dents naturelles (8), avec une véritable intégration naturelle des biomatériaux (9).

La biomimétique répond à une méthodologie bien précise, reposant sur trois points fondamentaux : l'identification d'un matériau du vivant présentant une propriété 
intéressante, sa compréhension sur la base de la connaissance de sa structure et de ses propriétés, puis le contre-typage de ce matériau utilisant des moyens généralement différents des moyens naturels. Il est pour cela important de connaître les spécificités propres des tissus de la dent naturelle, leur agencement (émail, dentine et jonction amélo-dentinaire), leur comportement optique et biomécanique (10), ainsi que les matériaux de restauration de ces tissus dentaires combinés aux systèmes CFAO.

L'émail et la dentine forment une structure composite conférant à la dent des caractéristiques singulières. L'émail est par essence l'enveloppe de l'organe dentaire, rigide, dure et cassante. Sa rigidité est de 84 $\mathrm{GPa}$, sa perte ayant une incidence directe sur son affaiblissement biomécanique. Il est donc primordial de préserver l'émail, car d'un point de vue fonctionnel, la dentine n'est rien sans sa coque (11).

La dentine est quand à elle capable d'absorber l'énergie d'une force et de se déformer sans fracture grâce à sa résilience et sa flexibilité. Sa rigidité, inférieure à celle de l'émail, est de $19 \mathrm{GPa}$. Les deux substrats de modules différents sont en contact grâce à des fibres de collagène, permettant un transfert de contraintes à cette interface, constituant ainsi un mécanisme efficace contre la propagation des fissures et l'absorption des contraintes (12) : c'est la jonction amélo-dentinaire (JAD). Cette interface structurelle et fonctionnelle entre un tissu extrêmement rigide et un tissu flexible donne à l'organe dentaire cette aptitude à supporter les contraintes masticatoires et thermiques tout au long de la vie (13), créant ainsi un excellent compromis entre rigidité, solidité et résilience.

Ces qualités décrites font de la JAD un modèle de référence pour les systèmes adhésifs utilisés pour renforcer l'intégrité biomécanique de la couronne dentaire (9). Les progrès réalisés par les systèmes adhésifs et les matériaux de restauration esthétiques nous permettent aujourd'hui de reproduire partielle-

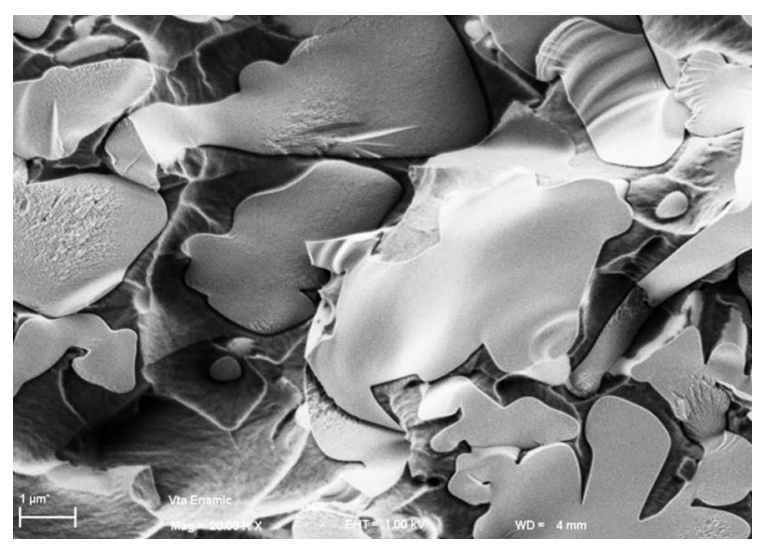

ment et modestement ce complexe amélo-dentinaire (14). Lors du protocole d'adhésion, les fractions inorganiques de la dentine sont dissoutes à l'acide orthophosphorique et le collagène mis à nu. Il est ensuite imprégné par l'adhésif. Cette couche de dentine préalablement traitée et infiltrée est appelée couche hybride. Cette dernière faite de tissus dentinaires et de résine adhésive intimement liés joue grandement sur la résistance du collage dentinaire (15). Bioémuler la liaison biologique entre l'émail et la dentine par cette couche hybride a donc pour objectif de stopper la propagation des fissures au niveau de la dentine et d'éviter une fracture coronaire.

Les matériaux de restauration esthétiques nous permettent également de nous rapprocher des caractéristiques mécaniques de l'émail et de la dentine.

L'émail doit être idéalement restauré par un matériau à haut module d'élasticité afin de restituer la rigidité de la couronne. La céramique est le matériau de choix car sa rigidité et sa résistance à la compression sont proches des valeurs de l'émail (7) (16).

De nouveaux types de matériau sont apparus sur le marché de la CFAO ces derniers mois, appelés matériaux hybrides ou céramiques hybrides, du fait de leur double composition. Ils sont présentés comme des matériaux composés de deux phases interpénétrées, un réseau de céramique et un réseau polymérique de résine composite. Ces matériaux hybrides combineraient à la fois les avantages mécaniques des céramiques et des résines composites avec:

$\checkmark$ une meilleure résistance à la propagation des fissures ;

plus de flexibilité (module de Young proche de celui de la dentine) ;

moins d'usure de l'antagoniste ;

$\checkmark$ une dureté proche des valeurs dentinaires ;

$\checkmark$ une résistance à l'abrasion proche de l'émail ;

\une usinabilité plus rapide.

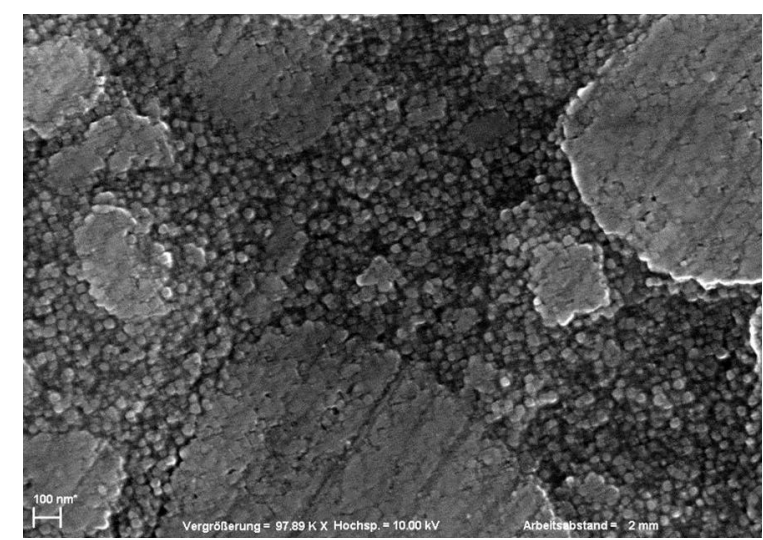

$\triangle$ Fig. 2 : Détail de la composition des deux matériaux hybrides au microscope électronique à balayage.

(a) Structure de l'Enamic (Vita) où l'on peut observer le réseau de céramique feldspathique (structures macroscopiques) infiltré par un polymère de composite.

(b) Structure du Lava Ultimate $(3 \mathrm{M})$ où l'on peut déceler les particules de céramique agglomérées.

(Source : Document interne 3M ESPE). 
Il existe deux matériaux hybrides présents sur le marché actuellement : le Lava Ultimate (3M ESPE), décrit comme un «composite hybride » et l'Enamic (Vita Zahnfabrik), décrit comme "une céramique hybride». Si le terme «hybride» est employé par les deux fabricants, il porte en revanche sur des aspects différents pouvant porter à confusion. En effet, d'après Le Petit Larousse, la définition du terme «hybride » est « qui est composé d'éléments disparates »; elle est donc proche du terme " composite» qui est « formé d'éléments divers et peu homogènes ». Qu'est-ce donc qu'un "composite hybride", si ce n'est un terme marketing utilisé par les fabricants? C'est pourquoi nous préfèrerons utiliser les vocables «composites techniques» et «céramiques techniques ", car mettant plus en valeur la technicité de ces matériaux par rapport à une céramique ou un composite traditionnel.

Le bloc Lava Ultimate de 3M ESPE est un matériau composé de particules de céramique dispersées enrobées dans une matrice de polymère. Ces particules de céramique représentent $80 \%$ du matériau pour ce qui est du poids. Les particules de céramique sont agglomérés en grappe et mesurent en moyenne $20 \mathrm{~nm}$ pour les particules de silice et $4-11 \mathrm{~nm}$ pour les particules de zircone (17). Comme pour une céramique classique, son assemblage fait appel aux techniques adhésives, mais le mordançage à l'acide fluorhydrique n'est plus nécessaire.

Le matériau Enamic de Vita Zahnfabrik présente quant à lui un réseau de vitrocéramique infiltré d'un polymère (RCIP). Contrairement à une céramique In Ceram, la phase vitreuse est remplacée ici par un polymère (18). Le réseau de céramique représente $86 \%$ du poids du matériau (en volume 70-75\%).

\section{CAS CLINIQUES}

Nous présentons 3 cas cliniques d'endocouronnes réalisées par CFAO directe à l'aide des 3 matériaux présentés, la céramique classique et les deux matériaux "hybrides ». La CFAO directe (lorsque l'enregistrement des données et l'usinage de la pièce prothétique sont réalisés au cabinet) permet la réalisation de la pièce prothétique en une seule séance, ce qui lui confère un intérêt biologique (étanchéité coronaire immédiate) et pratique, que ce soit pour le patient et le praticien.

Les étapes clés de la CFAO directe lors de la mise en œuvre de l'endocouronne sont résumées dans le tableau ci-dessous. Ces étapes sont, à quelques détails près, identiques pour les 3 types de matériaux.

\section{Endocouronne en céramique : cas clinique $\mathrm{n}^{\circ} 1$}

Une patiente de 41 ans se présente au cabinet pour une lésion carieuse volumineuse sur sa 16. La perte de substance de la 16 rentre dans les indications de réalisation d'endocouronne. Son délabrement est en effet supérieur aux $2 / 3$ de structure coronaire résiduelle. Pour ce cas clinique, le choix s'est porté sur un bloc céramique IPS Empress CAD de teinte A2 de faible translucidité, afin d'obtenir un meilleur rendu esthétique (21).

La dent est préparée en conservant un maximum de hauteur d'émail. L'empreinte optique de la préparation est par la suite réalisée directement en bouche. L'endocouronne est ensuite modélisée à l'aide du logiciel et des données qui ont été enregistrées. L'esthétique de la pièce prothétique est peaufinée, les points de contacts occlusaux et adjacents sont modifiés et validés.

\begin{tabular}{|c|c|c|}
\hline & $\begin{array}{l}\text { Lava Ultimate } \\
\text { (3M ESPE) }\end{array}$ & Enamic (VITA) \\
\hline Composition & $\begin{array}{c}\text { Charges : } \mathrm{SiO} 4, \\
\text { nanocluster de } \mathrm{SiO} 4 \\
\text { avec des nanomères } \\
\text { greffés, zircone } \\
\text { Monomères : NC }\end{array}$ & $\begin{array}{l}\text { Charges : Mark II } \\
\text { Monomères : UDMA }\end{array}$ \\
\hline Module d'élasticité (GPa) & $12,5-13$ & $30-37,95$ \\
\hline $\begin{array}{l}\text { Module de rupture en flexion } 3 \\
\text { points (MPa) }\end{array}$ & $200-225$ & $140-160$ \\
\hline Résistance à la compression ( $\mathrm{MPa})$ & 375 & 325 \\
\hline Dureté Vickers $\left(\mathrm{Kg} / \mathrm{mm}^{2}\right)$ & $100-105,9$ & $210-254,9$ \\
\hline Ténacité (MPa.m $\left.{ }^{1 / 2}\right)$ & $2-2,1$ & $1,5-1,7$ \\
\hline Module de Weibull & - & 20 \\
\hline
\end{tabular}

$\triangle$ Tableau I : Propriétés mécaniques et physiques des deux matériaux hybrides présents sur le marché (19) (20). Les valeurs présentées sont les valeurs extrêmes trouvées dans les différents articles de la littérature. Les données ne peuvent donc être comparées statistiquement entre elles du fait de la variabilité des méthodes et conditions de chaque test. 


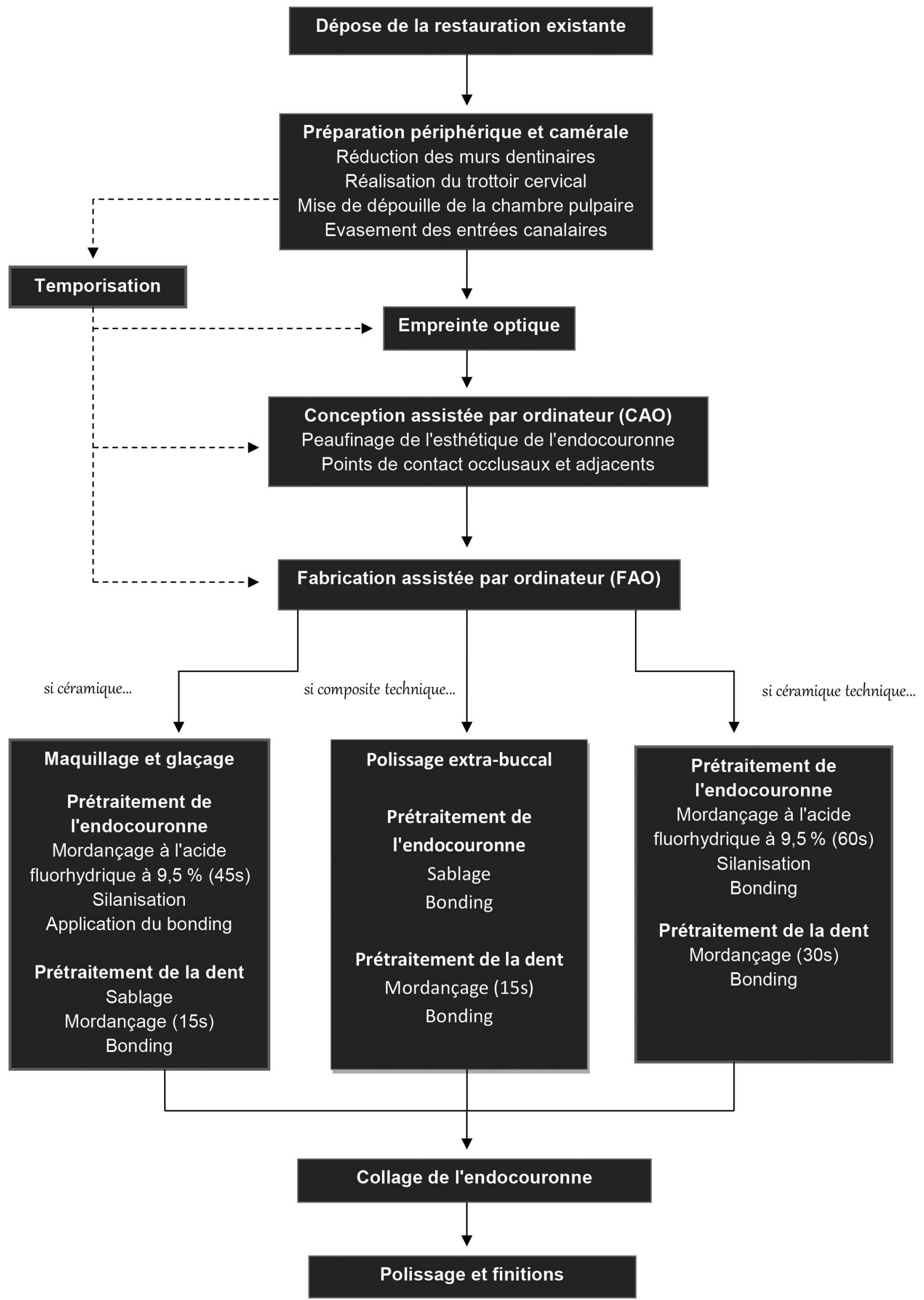

\footnotetext{
$\triangle$ Fig. 3 : Schéma récapitulatif de la mise en œuvre d'une endocouronne en céramique ou en composite.
} 
L'usinage de l'endocouronne par l'unité d'usinage du Cerec MC-XC dure environ 15 minutes. Après essayage en bouche, la pièce prothétique est maquillée et passée au four afin d'effectuer le glaçage de la céramique.

Afin d'optimiser les performances du collage de l'endocouronne, il est important de veiller au respect du protocole d'adhésion et au prétraitement des parois dentaires et de l'intrados. La préparation juxta-gingivale ne nous permet pas de poser le champ opératoire pour le collage. En revanche, l'utilisation simultanée de cotons salivaires et d'une aspiration efficace permet tout de même de poser l'endocouronne dans de bonnes conditions. Un composite de collage de type dual est utilisé. Après vérification finale de l'occlusion, on effectue le polissage de l'endocouronne.
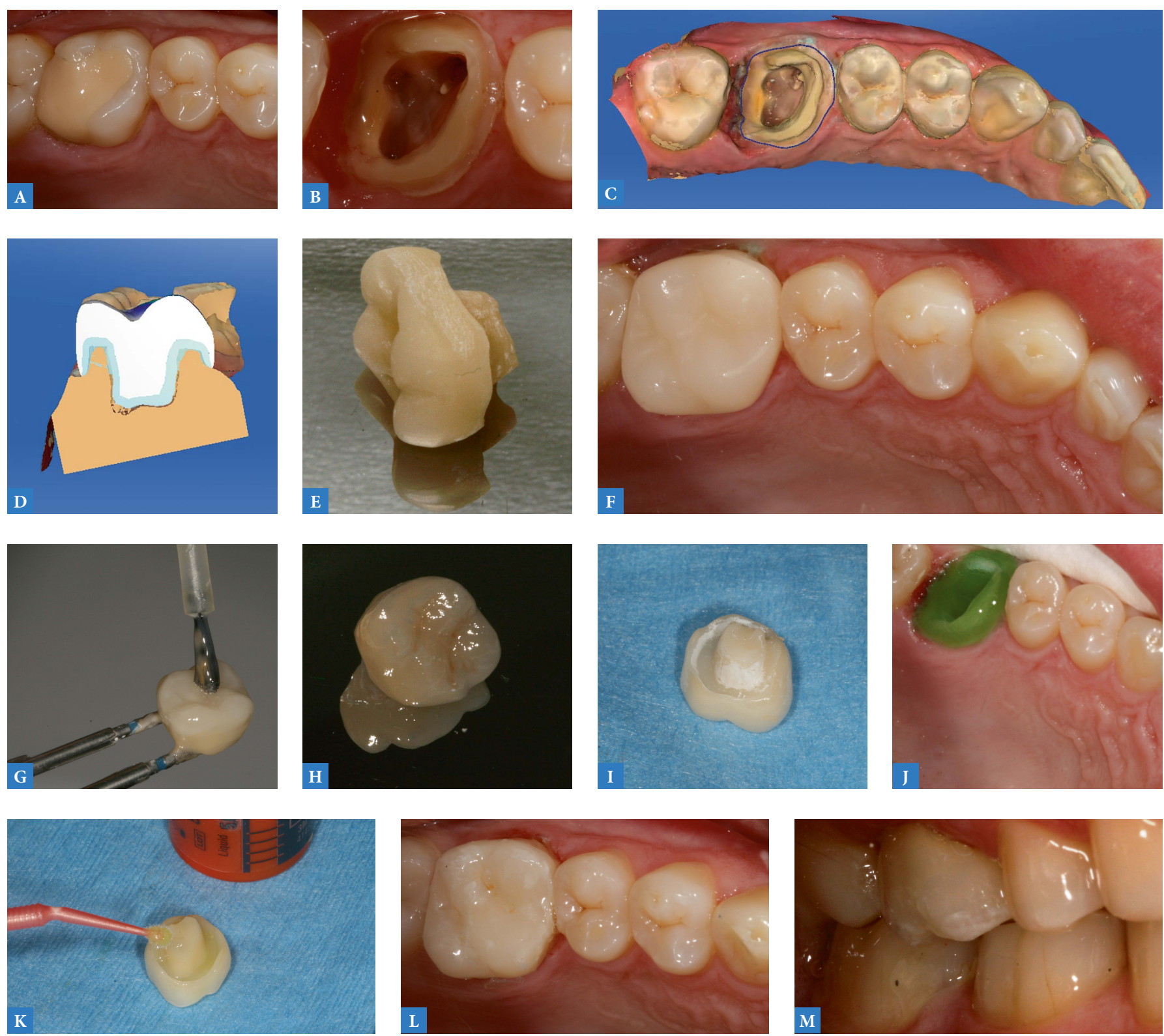

$\triangle$ Fig. 4 : Mise en œuvre de l'endocouronne en céramique.

(A) Situation initiale de la molaire maxillaire : vue occlusale

(B) Vue occlusale de la préparation coronaire et camérale pour endocouronne.

(C) Image modélisée de l'hémi-arcade 1.

(D) CAO : mise en évidence de la profondeur d'ancrage de l'endocouronne dans la chambre pulpaire et de l'épaisseur de céramique.

(E) Endocouronne après usinage.

(F) Essayage de l'endocouronne après usinage.

(G) Maquillage de la pièce prothétique.

(H) Endocouronne après glaçage de la céramique.

(I) Aspect crayeux et blanchâtre de l'intrados après mordançage à l'acide fluorhydrique à 9,5\%.

(J) Mordançage des tissus dentaires à l'acide orthophosphorique à $37 \%$.

(K) Application d'une couche d'adhésif non polymérisée sur l'intrados de l'endocouronne.

(L) (M) Vue occlusale et vestibulaire en OIM de l'endocouronne après polissage et réglage final de l'occlusion. 


\section{Endocouronne en technique céramique : cas clinique $n^{\circ} 2$}

Un patient âgé de 45 ans se présente au cabinet pour une couronne défectueuse sur la 16. L'indication d'endocouronne est posée en raison de parois supra-gingivales ainsi que d'un bandeau d'émail suffisant pour un collage efficace. Pour ce cas clinique, le choix s'est porté sur un bloc Enamic (Vita) de teinte M2 et de faible translucidité (LT).
L'usinage de l'endocouronne Enamic est plus rapide que celui de l'endocouronne en céramique feldspathique et dure environ 13 minutes. L'endocouronne en céramique hybride ne doit pas être cuite au four comme l'endocouronne en céramique feldspathique, ce qui constitue un gain de temps clinique non négligeable.
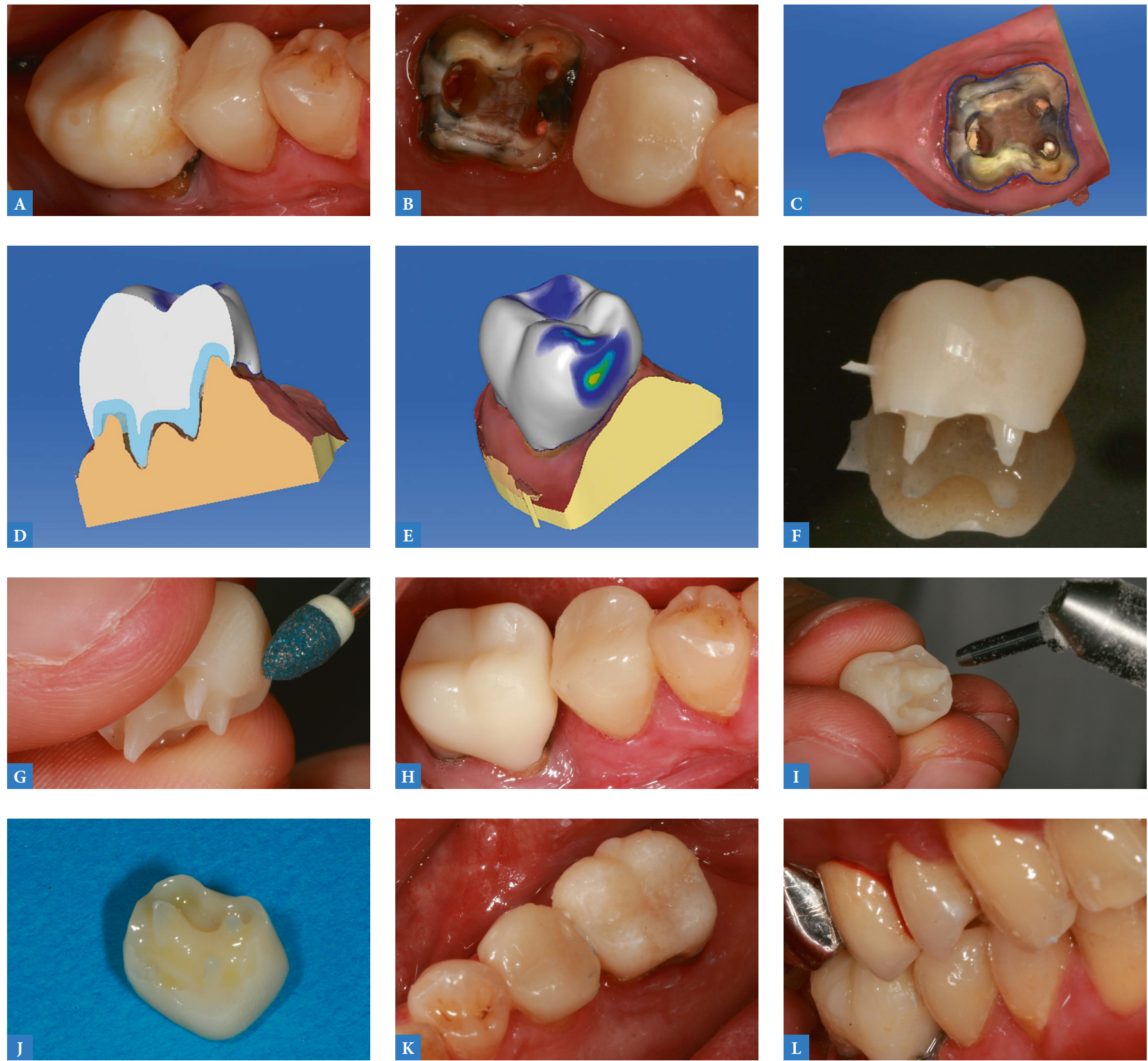

$\triangle$ Fig. 5 : Mise en œuvre de l'endocouronne en composite technique.

(A) Situation initiale de la molaire mandibulaire avant préparation pour endocouronne : vue occlusale.

(B) Vue occlusale de la préparation coronaire et camérale pour endocouronne.

(C) Tracé de la ligne de contour de la future endocouronne.

(D) Coupe de l'endocouronne avec mise en évidence de la profondeur d'ancrage de l'endocouronne dans la chambre pulpaire et de l'épaisseur de matériau.

(E) Points de contact proximal et occlusaux de l'endocouronne

(F) Endocouronne après usinage.

(G) Polissage extra-buccal de l'endocouronne.

(H) Essaye de l'endocouronne avant collage.

(I) Sablage de l'intrados de l'endocouronne.

(J) Application de l'adhésif.

(K) (L) Vue occlusale et vestibulaire en OIM de l'endocouronne après polissage et réglage final de l'occlusion. 


\section{Endocouronne en technique composite: cas clinique ${ }^{\circ} 3$}

Un patient, âgé de 52 ans, se présente pour une parodontite apicale aigüe sur sa 46 . L'indication d'endocouronne est posée en raison de parois supra-gingivales ainsi que d'un bandeau d'émail suffisant pour un collage efficace. Pour ce cas clinique, le choix s'est porté sur un bloc Lava Ultimate (3M ESPE) de teinte A2 et de faible translucidité (LT).

Les étapes de préparation périphérique et camérale sont identiques à celles de l'endocouronne en céramique classique, tout comme l'étape de CAO. L'usinage de la pièce en Lava Ultimate est plus court, environ 10 minutes En revanche, l'endocouronne en composite hybride ne doit pas être cuite au four comme l'endocouronne en céramique feldspathique, ce qui constitue un gain de temps clinique non négligeable. La pièce prothétique est polie à l'aide de fraises en silicone et essayée en bouche. Elle est ensuite collée à l'aide d'un composite de collage de type dual.

Après vérification finale de l'occlusion, le polissage extra-buccal est complété avec les mêmes instruments que lors du polissage extra-buccal afin d'améliorer le rendu de surface. Des composites fluides de finition sont enfin appliqués au collet, sur les sillons et sur les pointes cuspidiennes pour une meilleure caractérisation anatomique de l'endocouronne.

Les matériaux «hybrides » permettent au praticien un gain de temps clinique non négligeable (pas de cuisson au four, ni de glaçage). En revanche, l'aspect esthétique des deux matériaux hybrides, Lava Ultimate et Enamic, est moins satisfaisant que celui de la céramique. Ce constat est confirmé par Mörmann
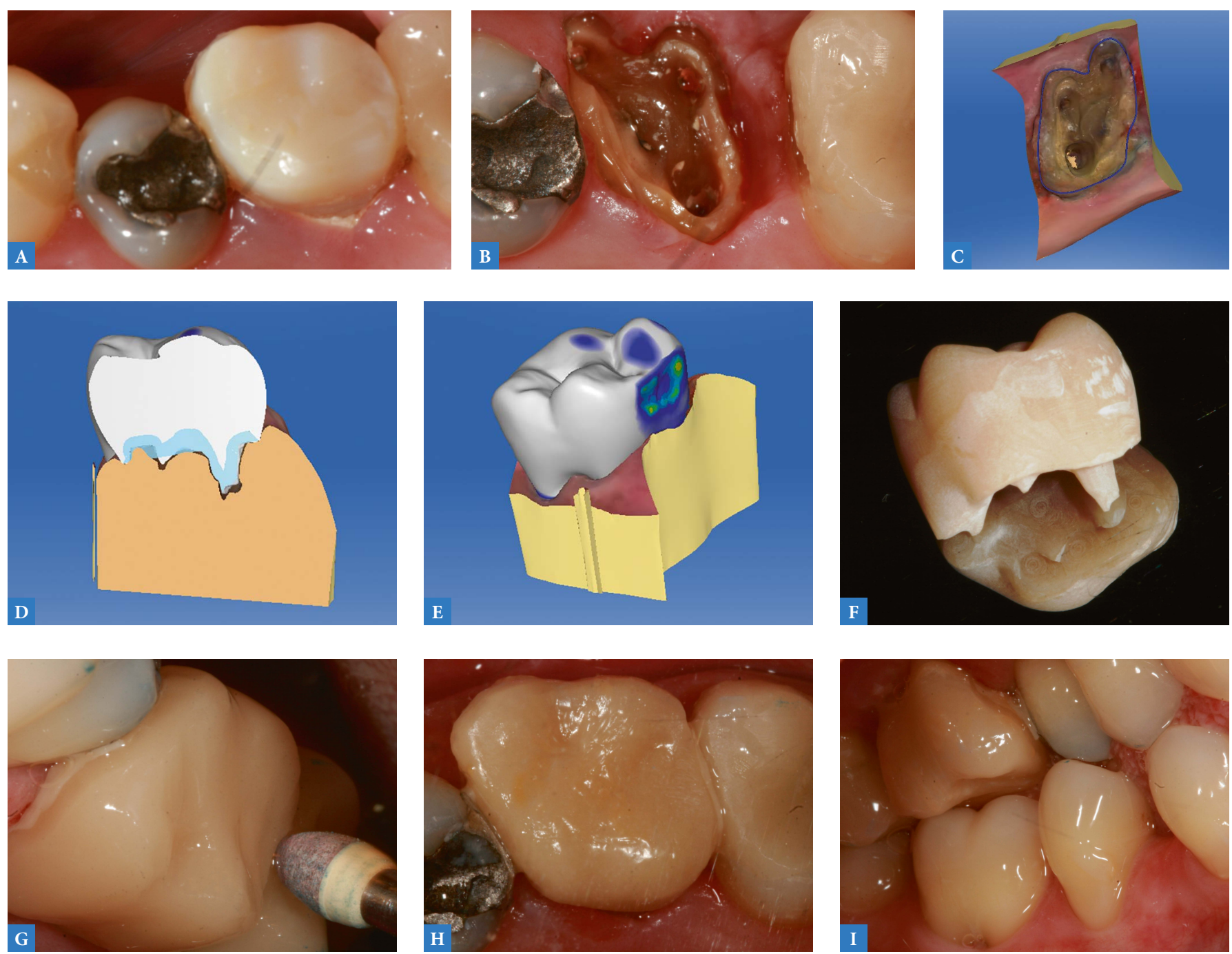

Fig. 6 : Mise en œuvre de l'endocouronne en céramique technique.

(A) Situation initiale de la molaire maxillaire. La dent a été préparée lors d'une séance précédente et protégée par une coiffe transitoire.

(B) Vue occlusale de la préparation périphérique et camérale pour endocouronne.

(C) Vue occlusale de la préparation modélisée et tracé de la ligne de contour.

(D) Coupe proximale de l'endocouronne et mise en évidence des ancrages radiculaires.

(E) Points de contact proximaux et occlusaux de l'endocouronne.

(F) Vue de la pièce prothétique après usinage.

(G) Essayage de l'endocouronne avant collage. (g) Polissage de la pièce prothétique à l'aide de pointes de polissage en silicone.

(H) (I) Vue occlusale et vestibulaire en OIM de l'endocouronne après polissage et réglage final de l'occlusion. 
WH et coll. qui comparent dans une étude in vitro la rugosité, l'usure, la brillance et la dureté de différents matériaux de restauration: zircone (inCoris TZI), céramique renforcée en disilicate de lithium (IPS e.max $\mathrm{CAD})$, céramique renforcée en leucite (Empress CAD), céramique feldspathque (VITA Mark II), céramique hybride (VITA Enamic) et nanocomposite hybride (Lava Ultimate). Si le comportement mécanique et l'usure des deux matériaux hybrides sont comparables aux valeurs obtenues par les céramiques, les matériaux hybrides présentent une brillance et un rendu esthétique moindre par rapport à toutes les céramiques étudiées (22).

Après la réalisation de ces trois cas cliniques, la céramique technique arrive en tête, suivi de la céramique feldspathique et du composite technique, en ce qui concerne la facilité de travail du matériau, de retouches et de polissage. Concernant l'esthétique en bouche, la céramique feldspathique reste le matériau de choix, suivi de la céramique technique et du composite technique.

\section{Endocouronne en 2 parties : utilisation combinée de céramique hybride et céramique classique : cas clinique $n^{\circ} 4$}

Nous avons réalisé sur une deuxième molaire mandibulaire une endocouronne composée en deux parties par CFAO directe. Bien que n'étant plus une reconstitution monobloc, les principes de préparation de l'endocouronne en deux parties restent identiques. Un moignon en céramique hybride (Enamic 1) comprenant l'ancrage caméral et canalaire est conçu dans un premier temps. Les étapes de conception et fabrication de la pièce prothétique sont strictement les mêmes que lors de l'endocouronne classique, à la différence près que la ligne de contour du moignon ne se situe pas sur la limite cervicale de la dent. Après son collage, la dent préparée est à nouveau scannée par la caméra intra-orale afin de réaliser la deuxième partie de l'endocouronne en céramique feldspathique renforcé en disilicate de lithium. Nous nous retrouvons donc avec une restauration faite de deux matériaux respectant au mieux les caractéristiques mécaniques des tissus dentaires.
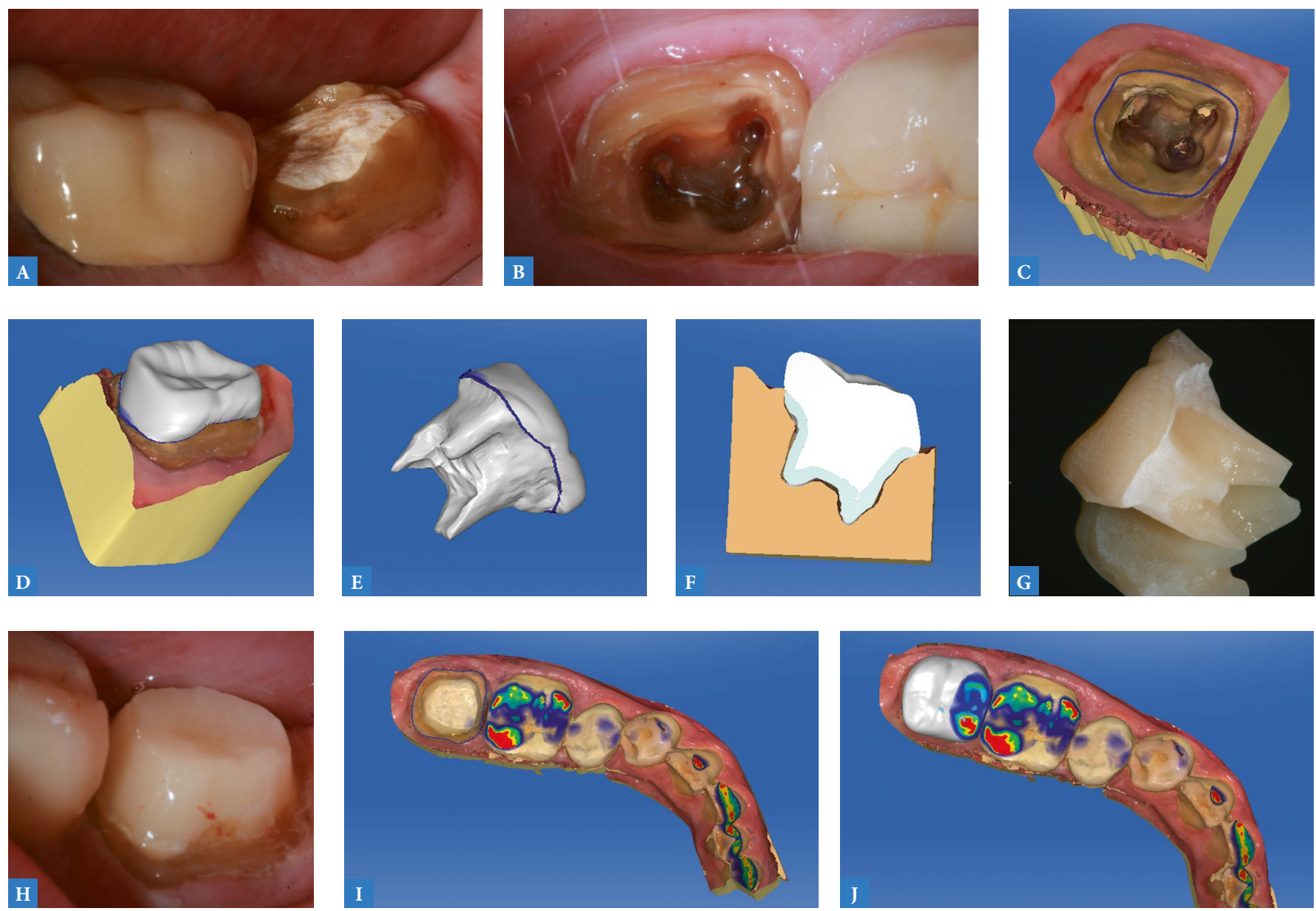

$\triangle$ Fig. 7 : Mise en œuvre de l'endocouronne bi-matière.

(A) Situation initiale.

(B) Vue occlusale de la molaire mandibulaire préparée.

(C) (D)(E) (F) Enregistrement et modélisation du moignon en céramique hybride.

$(\mathrm{G})$ Vue du moignon après usinage $(\mathrm{h})$ Vue vestibulaire du moignon après collage.

(I) (J) Enregistrement de la nouvelle situation et modélisation de la coiffe périphérique en céramique classique. 


\section{DISCUSSION}

En raison de son délabrement tissulaire souvent important et des conséquences biologiques et physico-chimiques engendrés, une dent traitée endodontiquement est moins résistante mécaniquement qu'une dent vitale. Lors de sa restauration, la démarche thérapeutique doit adopter une approche graduée selon le délabrement permettant de conserver le potentiel biologique de la dent dépulpée.

L'OMS propose actuellement aux pays développés de mettre en place une dentisterie dédiée au maintien durable des dents naturelles en bouche. La perennité des dents prévaut donc sur la notion de solidité maximales des restaurations. Au delà de sa résistance et de son invasivité, une restauration doit être évolutive et remplaçable tout au long de la vie du patient. Par conséquent, l'objectif est de concevoir des éléments prothétiques permettant à l'ensemble restauration dent d'avoir un comportement le plus naturel possible. De par sa préparation conservatrice préservant l'intégrité structurelle des racines et favorisant les techniques adhésives à une rétention macro-mécanique, les endocouronnes s'inscrivent définitivement dans cette démarche et dans la philosophie actuelle de la dentisterie contemporaine, à savoir les concepts d'invasivité minimale et de gradient thérapeutique.
Aujourd'hui, les matériaux de restauration développés nous permettent une approche biomimétique de la restauration des dents. La céramique classique est le matériau idéal pour le restauration de l'émail et l'un des avantages de la céramique hybride Enamic 1 et Enamic 2 en cours de développement est son module d'élasticité proche de la dentine (18-22 GPa), ce qui se traduit par des contraintes mieux réparties sur les structures dentaires. La céramique hybride (dentine) et la céramique classique (émail) seraient donc complémentaires, leur utilisation combinée permettrait de se rapprocher au mieux des propriétés des tissus de la dent naturelle.

\section{CONCLUSION}

L'endocouronne bi-matière présente un avantage mécanique par rapport à l'endocouronne classique, de par ses propriétés proches des tissus dentaires. Le moignon en céramique technique a également un avantage esthétique par rapport à un inlay-core, surtout si la coiffe périphérique est réalisée en tout-céramique. En revanche, le temps clinique est allongé par rapport à une endocouronne classique. Cette technique, encore expérimentale, doit encore faire ses preuves en ce qui concerne son comportement à long terme. 


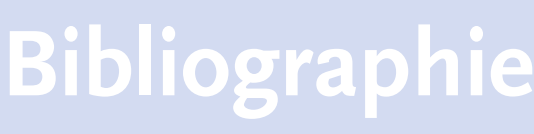

[1] Bindl A, Richter B \& Mörmann WH. Survival of Ceramic Computer-aided Design/ manufacturing Crowns Bonded to Preparations with Reduced Macroretention Geometry. The International Journal of Prosthodontics, 2005, 18 (3): 219-24.

[2] Lander E \& Dietschi D. Endocrowns: a Clinical Report. Quintessence International, 2008, 39 (2): 99-106.

[3] Dietschi D \& Spreafico R. Current Clinical Concepts for Adhesive Cementation of Toothcolored Posterior Restorations. Practical Periodontics and Aesthetic Dentistry: PPAD, 1998, 10 (1): 47-54; quiz 56.

[4] Roulet JF \& Degrange M. Collage et Adhésion : La Révolution Silencieuse. Quintessence International, 2000.

[5] Rocca GT \& Krejci I. Restaurations Adhésives Pour Dent Dépulpée. L'alternative Au Tout Couronne. Réalités Cliniques, 2011, 22 (1): 25-32.

[6] Magne P \& Douglas WH. Optimization of Resilience and Stress Distribution in Porcelain Veneers for the Treatment of Crownfractured Incisors. The International Journal of Periodontics \& Restorative Dentistry, 1999, 19 (6): 543-53.

[7] Magne P \& Belser UC. Porcelain Versus Composite Inlays/onlays: Effects of Mechanical Loads on Stress Distribution, Adhesion, and Crown Flexure. The International Journal of Periodontics \& Restorative Dentistry, 2003, 23 (6): 543-55
[8] Tirlet G \& Étienne O. La Biomimétique : Un Concept Contemporain Au Cour de La Dentisterie Adhésive. Conférence Au Congrès de l'Association Dentaire Française. 2013, November 28, Paris.

[9] Tirlet G \& Bazos P. La « Biomimétique » : Un Concept Contemporain Au Cœur de La Dentisterie Adhésive. Réalités Cliniques, 2013, 24 (4): 331-43.

[10] Magne P, Perakis N, Belser UC \& Krejci I. Stress Distribution of Inlay-anchored Adhesive Fixed Partial Dentures: a Finite Element Analysis of the Influence of Restorative Materials and Abutment Preparation Design. The Journal of Prosthetic Dentistry, 2002, 87 (5): 516-27.

[11] Bazos P \& Magne P. Bio-emulation: Biomimetically Emulating Nature Utilizing a Histo-anatomic Approach; Structural Analysis. The European Journal of Esthetic Dentistry: Official Journal of the European Academy of Esthetic Dentistry, 2011, 6 (1): 8-19.

[12] Ivancik J \& Arola DD. The Importance of Microstructural Variations on the Fracture Toughness of Human Dentin. Biomaterials, 2013, 34 (4): 864-74.

[13] Koubi S, Margossian P, Weisrok G, Lasserre J, Faucher A, Brouillet JL, Koubi G \& Tassery H. Restaurations Adhésives En Céramique : Une Nouvelle Référence Dans La Réhabilitation Du Sourire. Information Dentaire, 2009, 8: 363-74.

[14] Urabe I, Nakajima S, Sano H \& Tagami J. Physical Properties of the Dentin-enamel Junction Region. American Journal of Dentistry, 2000, 13 (3): 129-35.
[15] Lussi A \& Schaffner M. Evolutions En Odontologie Conservatrice. Paris : Quintessence International, 2013

[16] Reeh ES \& Ross GK. Tooth Stiffness with Composite Veneers: a Strain Gauge and Finite Element Evaluation. Dental Materials 1994, 10 (4): $247-52$.

[17] Zimmermann M, Mehl A \& Reich S. New CAD/CAM Materials and Blocks for Chairside Procedures. International Journal of Computerized Dentistry, 2013, 16 (2): 173-81.

[18] Kurbad A \& Kurbad S. A New, Hybrid Material for Minimally Invasive Restorations in Clinical Use. International Journal of Computerized Dentistry, 2013, 16 (1): 69-79.

[19] Dirxen C, Blunck U \& Preissner S. Clinical Performance of a New Biomimetic Double Network Material. The Open Dentistry Journal, 2013, 7: 118-22.

[20] Della Bona A, Corazza PH \& Zhang Y. Characterization of a Polymer-infiltrated Ceramic-network Material. Dental Materials, 2014 May ; 30 (5) : 564-9

[21] Cazier S, Chieze JB \& Hrycenko MA. Place de La CFAO Directe En Omnipratique. Inlay-Onlay. Réalités Cliniques, 2009, 20 (4): 219-30.

[22] Mörmann WH, Stawarczyk B, Ender A, Sener B, Attin T \& Mehl A. Wear Characteristics of Current Aesthetic Dental Restorative CAD/CAM Materials: Twobody Wear, Gloss Retention, Roughness and Martens Hardness. Journal of the Mechanical Behavior of Biomedical Materials, 2013, 20 (April): 113-25. 